This derivation of Theorem 2 from Theorem 1 was shown to us by C. T. C. Wall.

\title{
REFERENCES
}

1. S. Eilenberg and T. Ganea, On the Lusternik-Schnirelmann category of abstract groups, Ann. of Math. 65 (1957), 517-518.

2. A. G. Kurosh, The theory of groups, Transl. by K. A. Hirsch, Chelsea, New York, 1956.

3. J.-P. Serre, Sur la dimension cohomologique des groupes profinis, Topology 3 (1965), 413-420.

4. C. T. C. Wall, Poincare complexes. I, Ann. of Math. 86 (1967), 213-245.

University of California, Berkeley

\section{ON THE NORM OF STABLE MEASURES ${ }^{1}$}

BY LUDWIG ARNOLD AND JOHANNES MICHALICEK

Communicated by R. Creighton Buck, October 12, 1967

1. Limits of convolution powers and stable measures. Let $M(R)$ denote the Banach algebra of all complex-valued regular finite measures defined on the Borel sets of the real line $R$, where multiplication is defined by convolution, and

$$
\|\mu\|=\sup \sum\left|\mu\left(R_{i}\right)\right|,
$$

the supremum being taken over all finite collections of pairwise disjoint sets $R_{i}$ whose union is $R$. Let $B(R)$ be the set of all Fourier transforms of measures in $M(R)$.

In [1], we characterized all possible limits

$$
\lim _{n \rightarrow \infty}\left(\hat{\nu}\left(t / B_{n}\right)\right)^{n} \exp \left(i t A_{n}\right)=\hat{\mu}(t) \text { for all } t \neq 0,
$$

where $A_{n} \in R, B_{n}>0, \hat{\nu}, \hat{\mu} \in B(R)$. This is a generalization of an old problem in probability theory (see e.g. [4]). One can show that a measure $\mu$ appears as a limit if and only if it is stable, i.e. has the following property: For all $a>0, b>0$ there exist $c>0$ and $\gamma \in R$ such that

$$
\hat{\mu}(a t) \hat{\mu}(b t)=\hat{\mu}(c t) \exp (i \gamma t) \quad \text { for all } t \in R \text {. }
$$

${ }^{1}$ Sponsored by the Mathematics Research Center, United States Army, Madison, Wisconsin, under Contract No. DA-31-124-ARO-D-462. 
In other words, a stable measure convolved with itself reproduces itself after being properly shifted and scaled. Consequently, stable measures may be considered as a substitute for idempotent measures, which except for degenerate ones do not exist on the real line.

Besides the degenerate measure $\mu=0$ and $\mu=\delta_{\beta}$ (unit mass at $x=\beta$ ), a measure is a solution of (1) if and only if its Fourier transform is of the form

$$
\begin{aligned}
\hat{\mu}(t)=\exp \left(-c|t|^{\alpha}+i \beta t\right) & \text { for } t \geqq 0, \\
& =\exp \left(-d|t|^{\alpha}+i \beta t\right) \quad \text { for } t<0,
\end{aligned}
$$

or

$$
\begin{aligned}
\hat{\mu}(t) & =\exp (-c|t|+i \beta t \log |t|) & \text { for } t \geqq 0, \\
& =\exp (-d|t|+i \beta t \log |t|) & \text { for } t<0,
\end{aligned}
$$

where $\beta \in R, \alpha \in R, \alpha \neq 0 ; c$ and $d$ are complex constants with $\operatorname{Re}(c)>0$, $\operatorname{Re}(d)>0$. For $\alpha>0$ the corresponding measure $\mu$ is absolutely continuous; for $\alpha<0$ the measure $\delta_{\beta}-\mu$ is absolutely continuous.

2. Symmetric real-valued stable measures. By (1),

$$
\|\mu * \mu\|=\|\mu\|
$$

for every stable measure. Therefore, either $\|\mu\|=0$ or $\|\mu\| \geqq 1$. The stable probability measures clearly have $\|\mu\|=1$. One sees easily that $\|\mu\|>1$ for every stable measure which is not a probability measure. For $\alpha<0$ we even have $\|\mu\|>2$.

In this section we confine ourselves to

$$
\hat{\mu}_{\alpha}(t)=\exp \left(-|t|^{\alpha}\right), \quad \alpha \in R, \alpha \neq 0 .
$$

Clearly $\left\|\mu_{\alpha}\right\|$ is equal to 1 for $0<\alpha \leqq 2$, is bigger than 1 for $\alpha>2$, and is bigger than 2 for $\alpha<0$.

Our tool will be an approximation of $\hat{\mu}_{\alpha}$ by a function whose norm can be calculated, and the repeated use of

Lemma 1 (BeuRLing [2]). (i) Let $\phi$ be absolutely continuous and $\phi$, $\phi^{\prime} \in L_{2}(R)$. Then $\phi=\hat{\mu} \in B(R), \mu$ is absolutely continuous, and

$$
\|\mu\| \leqq\left(\int_{-\infty}^{\infty}|\phi(t)|^{2} d t \int_{-\infty}^{\infty}\left|\phi^{\prime}(t)\right|^{2} d t\right)^{1 / 4} .
$$

(ii) An even function $\phi$ is in $B(R)$ if $\phi(t) \rightarrow 0(t \rightarrow \infty)$ and if the integral below is convergent. Then, putting $\phi=\hat{\mu}$,

$$
\|\mu\| \leqq \int_{0}^{\infty} t\left|d \phi^{\prime}(t)\right| .
$$


TheOREM 1. (i) For $\alpha<0$,

$$
2<\left\|\mu_{\alpha}\right\| \leqq 2+\left((2 \alpha(\alpha-1))^{1 / 2}-\alpha\right) \exp (1 / \alpha-1) .
$$

(ii) If $\alpha \beta>0$ then

$$
\left\|\mu_{\alpha}-\mu_{\beta}\right\| \leqq|\beta-\alpha| K(\alpha, \beta),
$$

where $K$ is locally bounded.

Proof. (i) The inflection points of $\hat{\mu}_{\alpha}$ are $\pm t_{0}$ where $t_{0}=((\alpha-1) / \alpha)^{1 / \alpha}$. Approximate $1-\hat{\mu}_{\alpha}$ by

$$
\begin{aligned}
g_{\alpha}(t) & =1-\hat{\mu}_{\alpha}(t) \quad \text { for }|t|>t_{0}, \\
& =1+\hat{\mu}_{\alpha}^{\prime}\left(-t_{0}\right)\left(t+t_{0}\right)-\hat{\mu}_{\alpha}\left(-t_{0}\right) \quad \text { for }-t_{0} \leqq t<0, \\
& =1+\hat{\mu}_{\alpha}^{\prime}\left(t_{0}\right)\left(t-t_{0}\right)-\hat{\mu}_{\alpha}\left(t_{0}\right) \quad \text { for } 0 \leqq t \leqq t_{0} .
\end{aligned}
$$

The function $g_{\alpha}$ is even and concave in $(0, \infty)$, and therefore by Polya's criterion is positive definite. Thus $g_{\alpha}=\hat{\nu}_{\alpha}$ where $\left\|\nu_{\alpha}\right\|=g_{\alpha}(0)$ $=1-\exp (1 / \alpha-1)$. For the remainder $\hat{\nu}_{\alpha}-\left(1-\hat{\mu}_{\alpha}\right)$, we find by Lemma 1 (i)

$$
\left\|\nu_{\alpha}-\left(\delta_{0}-\mu_{\alpha}\right)\right\| \leqq(2 \alpha(\alpha-1))^{1 / 2} \exp (1 / \alpha-1) .
$$

(ii) Lemma 1 (ii) yields

$$
\left\|\hat{\mu}_{\alpha}-\hat{\mu}_{\beta}\right\| \leqq \int_{0}^{\infty} t\left|\hat{\mu}_{\beta}^{\prime \prime}(t)-\hat{\mu}_{\alpha}^{\prime \prime}(t)\right| d t
$$

By the mean value theorem applied to the variable $\alpha$,

$$
\hat{\mu}_{\beta}^{\prime \prime}(t)-\hat{\mu}_{\alpha}^{\prime \prime}(t)=\left.(\beta-\alpha) \frac{\partial \hat{\mu}_{\gamma}^{\prime \prime}(t)}{\partial \gamma}\right|_{\gamma=\alpha+(\beta-\alpha) \theta}
$$

$0<\theta<1$. An elementary calculation yields

$$
\int_{0}^{\infty} t\left|\frac{\partial \hat{\mu}_{\gamma}^{\prime \prime}(t)}{\partial \gamma}\right| d t \leqq K(\alpha, \beta),
$$

where $K$ is locally bounded.

Corollary 1. (i) $\lim _{\alpha \rightarrow-0}\left\|\mu_{\alpha}\right\|=2$.

(ii) The function $\alpha \rightarrow \mu_{\alpha}$ mapping $R-\{0\}$ into $M(R)$ is continuous with respect to the norm topology in $M(R)$.

It should be mentioned that if we define $\mu_{0}=e^{-1} \delta_{0}$, then $\mu_{\alpha}$ is continuous at $\alpha=0$ in the weak* topology of $M(R)$.

3. Asymptotic behavior of $\left\|\mu_{\alpha}\right\|$. 
Theorem 2. $A s|\alpha| \rightarrow \infty$,

$$
\left\|\mu_{\alpha}\right\|=\left(4 / \pi^{2}\right) \log |\alpha|+O(1) .
$$

For the proof of this fact we need the following

Lemma 2. Consider the trapezoid-shaped function

$$
\begin{aligned}
\hat{\nu}_{a, b}(t) & =0 & & \text { for }|t| \geqq b, \\
& =(1 / a)(b-|t|)) & & \text { for } b-a \leqq|t|<b, \\
& =1 & & \text { for }|t|<b-a,
\end{aligned}
$$

where $b>a>0$. Then, for $b / a \rightarrow \infty$,

$$
\left\|\nu_{a, b}\right\|=\left(4 / \pi^{2}\right) \log (b / a)+O(1) .
$$

For the proof write $\hat{\nu}_{a, b}=\hat{\sigma}_{1}+\hat{\sigma}_{2}$, where

$$
\hat{\sigma}_{1}(t)=\sum_{|k| \leq\{b / a]-1} \hat{\rho}(t+k a)
$$

and

$$
\begin{aligned}
\hat{\rho}(t) & =1-|t| / a & & \text { for }|t| \leqq a, \\
& =0 & & \text { for }|t|>a .
\end{aligned}
$$

Lemma 1 (i) applied to $\sigma_{2}=\nu_{a, b}-\sigma_{1}$ yields $\left\|\sigma_{2}\right\| \leqq 2$. Furthermore, by direct calculation using Poisson's summation formula, we obtain

$$
\left\|\sigma_{1}\right\|=(1 / \pi) \int_{-\pi}^{\pi}\left|D_{[b / a]-1}(x)\right| d x,
$$

where $D_{n}$ is the Dirichlet kernel.

Proof of Theorem 2. Assume $\alpha \geqq 1$. Approximate $\hat{\mu}_{\alpha}$ by a trapezoid $\hat{\nu}_{\alpha}$ so that its sides coincide with the tangents at the inflection points of $\hat{\mu}_{\alpha}$. This leads to $b / a=\alpha \exp (1 / \alpha-1)$, and by the above lemma for $\alpha \rightarrow \infty$,

$$
\left\|\nu_{\alpha}\right\|=\left(4 / \pi^{2}\right) \log \alpha+O(1) .
$$

Again by Lemma 1 (i), $\left|\left\|\nu_{\alpha}\right\|-\left\|\mu_{\alpha}\right\|\right|=O(1)$. We proceed similarly for $\alpha>0$.

In the same way we can show that $\left\|\mu_{\alpha}+\mu_{-\alpha}\right\|=O(1)$. Although $\left\|\mu_{\alpha}\right\| \rightarrow \infty$ we have for the densities $g_{\alpha}$ of $\mu_{\alpha}$

$$
g_{\alpha}(x) \rightarrow(\sin x) / \pi x \quad \text { as } \alpha \rightarrow \infty
$$

in the norm of $L_{2}(R)$, as Parseval's equation shows. 
Corollary 2. For every $\epsilon>0$ there is a $\mu \in M(R)$ such that $\|\mu\|=1$, but $\|\mu * \mu\|<\epsilon$.

To see this, choose $\mu_{\alpha}$ such that $\left\|\mu_{\alpha}\right\|>\epsilon^{-1}$. Now take $\mu=\mu_{\alpha} /\left\|\mu_{\alpha}\right\|$ and use (2).

Corollary 2 is true also in $M(G)$, where $G$ is the circle group or any compact connected abelian group, since in such a group there exist idempotent measures with arbitrarily large norm. See Cohen [3].

4. A skew case. Consider the stable measures $\mu_{c, \alpha}$ corresponding to

$$
\begin{aligned}
\hat{\mu}_{c, \alpha}(t) & =\exp \left(-c|t|^{\alpha}\right) & \text { for } t \geqq 0, \\
& =\exp \left(-|t|^{\alpha}\right) & \text { for } t<0,
\end{aligned}
$$

where $\alpha \notin(0,1)$ and $c \in R$.

Theorem 3. For $c \rightarrow \infty$,

$2 \log c+O(1) \leqq\left\|\mu_{c, \alpha}\right\| \leqq 2|2 \alpha \exp (1 / \alpha-1)-1| \log c+O(1)$.

A technique similar to the one used in Theorem 2 leads to the conjugate Fejér kernel rather than to the Dirichlet kernel.

\section{REFERENCES}

1. L. Arnold and J. Michalicek, Complex-valued stable measures and their domains of attraction, Math. Research Center Tech. Summary Rep. No. 805, 1967.

2. A. Beurling, Sur les intégrales de Fourier absolument convergentes et leur application à une transformation fonctionelle, Neuvième Congrès des Mathematiciens Scandinaves, 1938, pp. 345-366.

3. P. J. Cohen, On a conjecture of Littlewood and idempotent measures, Amer. J. Math. 82 (1960), 191-212.

4. B. W. Gnedenko and A. N. Kolmogorov, Grenzverteilungen von Summen unabhängiger Zufallsgrössen, Akademie-Verlag, Berlin, 1959.

Mathematics Research Center, University of Wisconsin 